Background It has been reported that the determination of magnesium levels could be used as a surrogate marker of efficacy in chemotherapy regimens with cetuximab.

Purpose To investigate the hypomagnesemia caused by cetuximab as a predictor of efficacy and outcome in patients affected by head and neck cancer in first-line treatment.

Materials and Methods Retrospective observational study (Study period: November 2008-October 2012). We analysed patients with head and neck carcinoma treated with cetuximab in first-line treatment, who had magnesium determinations from the start of treatment until one month after the end of treatment with cetuximab. Patients with magnesium determinations were stratified into two groups: Patients who presented hypomagnesemia during the treatment $(<1.7 \mathrm{mg} / \mathrm{dL})$ and patients who didn't present hypomagnesemia. The primary outcome was to compare remission rate, progression-free survival (PFS) and overall survival (OS) in the two groups. PFS and OS were both determined by the Kaplan-Meier product-limit method.

Results We collected a total of 14 patients (92.8\% male). The median age at onset of treatment was 61 years (range: $21-86$ ). Six patients developed hypomagnesemia during treatment. The most common diagnosis was carcinoma of the oral cavity $(28.6 \%)$ followed by laryngeal carcinoma (21.4\%). The group of patients who presented hypomagnesemia showed a higher remission rate $(66.7 \%$ vs. $37.5 \%$ patients), OS [mean: 34.8 (18.8 to 50.9$)$ ) vs. 22.4 (95\% CI: 11.9 to 32.9 months, $p=0.532$ ] and PFS [34.5 months (18.11 to 50.9), vs. 19.7 (7.8-31.5) $\mathrm{p}=0.456$ ] in comparison with the group in which hypomagnesaemia was not detected.

Conclusions Despite the small number of patients studied, hypomagnesemia could be a marker of cetuximab efficacy in first-line treatment in patients with head and neck cancer. Magnesium levels should be determined routinely in patients treated with cetuximab.

No conflict of interest.

\section{DGI-043 INCREASED COST OF ERYTHROPOIESIS-STIMULATING AGENTS IN SOME SPECIAL SITUATIONS}

doi:10.1136/ejhpharm-2013-000276.309

'V Escudero-Vilaplana, 2JM López-Gómez, ${ }^{2} \mathrm{~A}$ Vega-Martínez, 'A Ais-Larisgoitia, 'B Marzal-Alfaro, 'I Yeste-Gómez, 'A Ribed-Sánchez, 'P Arrabal-Durán, 'A GiménezManzorro, 'M Sanjurjo-Sáez. 'Hospital General Universitario Gregorio Marañón, Pharmacy, Madrid, Spain; ${ }^{2 H}$ ospital General Universitario Gregorio Marañón, Nephrology, Madrid, Spain

Background Situations such as a previous kidney transplant or dialysis may increase the dose requirement of erythropoiesisstimulating agents (ESAs), which is associated with a higher cost.

Purpose To examine the difference in cost between the use of ESAs for pre-dialysis and dialysis (peritoneal dialysis or haemodialysis) patients and for patients with or without a kidney transplant.

Materials and Methods A descriptive, transversal study was carried out in patients treated with ESAs for anaemia secondary to chronic kidney disease in a tertiary hospital over a month in 2011. ESAs used were: epoetin ( $\alpha$ or $\beta$ ), darbepoetin $\alpha$ and continuous erythropoietin receptor activator (CERA). The principal variable was patient-month cost, calculated as the cost of the dose unit for each ESA type (IU or mcg) multiplied by the monthly dose per patient.

Results 333 patients were included. $26.2 \%$ had previously had a kidney transplant (10.3\% epoetin, 33.3\% darbepoetin $\alpha, 56.3 \%$ CERA). Median [p25, p75] patient-month cost for patients with kidney transplant vs. patients who had not had a kidney transplant was: epoetin (191.3 [95.6, 414.5] euros vs. 103.2 [63.8, 191.3] euros, $p=0.060)$, darbepoetin $\alpha(144.0[72.0,288.0]$ euros vs. 144.0
$[72.0,216.0]$ euros, $p=0.136)$ and CERA $(196.7$ [172.1, 295.0] euros vs. $98.3[59.0,147.5]$ euros, $\mathrm{p}<0.001)$.

$30.9 \%$ patients were on dialysis $(35.0 \%$ epoetin, $58.3 \%$ darbepoetin $\alpha, 6.8 \%$ CERA). Median [p25, p75] patient-month cost for patients on dialysis vs. not yet on dialysis was: epoetin (151.1 [74.1, 239.1] euros vs. $92.1[59.5,165.6]$ euros, $p=0.006)$, darbepoetin $\alpha$ (144.0 [72.0, 216.0] euros vs. 144.0 [67.2, 229.2] euros, $p=0.888)$ and CERA (393.4 [98.3, 491.7] euros vs. 147.5 [98.3, 196.7] euros, $\mathrm{p}=0.035$ )

Conclusions The cost of epoetin and CERA is greater for both patients with a kidney transplant and patients on dialysis. However there was no difference regarding darbepoetin $\alpha$.

No conflict of interest.

\section{DGI-044 INHALED COLISTIN IN TREATMENT OF CHRONIC COLONISATION PSEUDOMONAS AERUGINOSA IN PATIENTS WITH NON-CYSTIC FIBROSIS BRONCHIECTASIS OR CHRONIC OBSTRUCTIVE PULMONARY DISEASE}

doi:10.1136/ejhpharm-2013-000276.310

B López Virtanen, S Vázquez Troche, J Valdueza Beneitez, B Nogal Fernández M Noguerol Cal, M Rodríguez María. Hospital El Bierzo, Pharmacy, Ponferrada, Spain

Background Chronic bronchial infection with Pseudomonas aeruginosa in patients with non-cystic fibrosis (CF) bronchiectasis/ chronic obstructive pulmonary disease (COPD) is related to worsening lung function and increased morbidity and mortality. Inhaled antibiotics represent an effective therapeutic approach for these diseases.

Purpose To evaluate the use of inhaled colistin in the treatment of chronic colonisation with Pseudomonas aeruginosa in patients with non-CF bronchiectasis/COPD.

Materials and Methods Retrospective study of patients with $\mathrm{COPD} /$ non-CF bronchiectasis colonised with Pseudomonas aeruginosa treated with inhaled colistin for at least three months from January 2008 to April 2012. Data collected: sex, age, diagnosis, duration of the treatment, disease-related hospitalizations pre and post-treatment, sputum cultures, clinical evolution.

Results 5 patients ( 3 with non-CF bronchiectasis and 2 with COPD) and 6 treatment episodes ( 1 patient received 2 courses of treatment) were included. Treatment duration was 27.6 months (range 4-48). Average cost per patient $€ 13,896$ (range $€ 2,950$ $25,888)$. In 5 episodes, treatment was initiated after $\geq 4$ consecutive sputum cultures positive for Pseudomonas resistant to tobramycin/ciprofloxacin. No difference in number of diseaserelated hospitalizations/month pre-and post-treatment ( 0.25 vs. 0.26). Sputum Pseudomonas eradication (3 consecutive negative sputum samples) was reported in 2 patients; treatment was continued, which was an unnecessary cost of $€ 15,500$ (22\% of total costs). No resistance developed to colistin. In two episodes (one with eradication) clinical improvement occurred (reduction in cough and expectoration).

The number of hospitalizations/month was similar before and after treatment, and the microbiological response (negative results on sputum) and the clinical response (reducing cough and sputum purulence) was moderate (2 of 6 episodes).

Three patients died from their bronchial disease.

Conclusions In most episodes the initial prescription was correct ( $\geq 3$ consecutive sputum cultures positive).

In patients whose Pseudomonas had been eradicated, treatment was continued, therefore sputum cultures should be monitored more frequently.

No effective treatment was observed.

No conflict of interest. 